\title{
Persistent Thrombocytopenia Following Dengue Shock Syndrome
}

\author{
Utkarsh Kohli, Sunil Saharan, Rakesh Lodha and S.K. Kabra \\ Department of Pediatrics, All India Institute of Medical Sciences, Ansari Nagar, New Delhi, India
}

\begin{abstract}
Though thrombocytopenia is one of the hallmarks of dengue hemorrhagic fever/ dengue shock syndrome, persistence of the same is rare. We report an 11 year-old child with dengue shock syndrome, who developed persistent thrombocytopenia. The possible mechanisms are discussed. [Indian J Pediatr 2008; 75 (1) : 82-83] E-mail: rakesh_lodha@ @otmail.com
\end{abstract}

Key words : Thrombocytopenia; Dengue shock syndrome

Thrombocytopenia is one of the hallmarks of dengue hemorrhagic fever (DHF), ${ }^{1}$ but platelet counts usually recover during convalescence. ${ }^{2}$ However, prolonged thrombocytopenia has rarely been reported in dengue patients. ${ }^{3,4}$ We report an eleven-year-old male child with immune mediated and corticosteroid unresponsive, prolonged thrombocytopenia following dengue shock syndrome.

\section{CASE REPORT}

This eleven-year-old male child, a resident of Delhi, presented with fever for 4 days, vomiting for 3 days and epistaxis for 1 day. The child developed a diffuse erythematous maculopapular rash on day 2 of fever which lasted for $24 \mathrm{hr}$. There was no history of pain abdomen, sore throat, respiratory distress, diarrhea, jaundice, altered sensorium or bleeding from any other site. On examination, the patient was febrile, had heart rate $80 / \mathrm{min}$ and hypotension (Blood pressure $80 / 60 \mathrm{~mm}$ $\mathrm{Hg}$ ). Tourniquet test (Hess test) was positive. Chest examination revealed decreased air entry in bilateral infra-axillary region and liver was palpable $2 \mathrm{~cm}$ below costal margin (span $8 \mathrm{~cm}$ ) on abdominal examination. Cardiovascular and neurological examinations were normal.

Initial investigations revealed a hematocrit of $50 \%$, platelet count of $30,000 / \mathrm{mm}^{3}$ and total leucocyte count

Correspondence and Reprint requests : Dr Rakesh Lodha, Assistant Professor, Department of Pediatrics, All India Institute of Medical Sciences, Ansari Nagar, New Delhi-110029, India.

[Received December 22, 2006; Accepted October 31, 2007]
(TLC) of $4500 / \mathrm{mm}^{3}$. Prothrombin time, renal, and liver function tests were normal. Dengue spot test (Bioline one step rapid immunochromatographic test; Standard diagnostics, Kyonggi-do, Korea) for anti-dengue IgG and IgM antibodies was positive. Disseminated intravascular coagulation (DIC) profile at admission was negative; chest radiograph showed bilateral pleural effusion.

Based on clinical findings and laboratory investigations, diagnosis of dengue shock syndrome was made and the patient was started on Ringers lactate at the rate of $15 \mathrm{ml} / \mathrm{Kg} / \mathrm{hr}$ as per standard treatment guidelines. ${ }^{5}$ The patient responded to fluid bolus with improvement in blood pressure and subsequently, fluid infusion rate was gradually dropped to $7 \mathrm{ml} / \mathrm{Kg} / \mathrm{hr}$ after $24 \mathrm{hrs}$ of initiation of therapy. On Day 2 of admission the patient developed significant respiratory distress due to increase in bilateral pleural effusion and ascites, and this necessitated intubation and mechanical ventilation. Platelet counts also dropped to $17,000 / \mathrm{mm}^{3}$ and he was transfused with 3 units of platelet rich plasma (PRP). Capillary leak resolved after $72 \mathrm{hrs}$ but thrombocytopenia persisted and the patient required daily PRP transfusions. The hospital course was further complicated by pneumonia, and subsequently, by acute respiratory distress syndrome (ARDS). The child responded to intravenous antimicrobials, followed by intravenous corticosteroids, and was weaned off the ventilator after 4 wks. During this period of 4 week, the thrombocytopenia persisted and the child received a total of 50 units of PRP to maintain the platelet counts above $40,000 / \mathrm{mm}^{3}$; multiple DIC profiles and blood cultures were negative. Bone marrow biopsy revealed normalcy of all three cell lines. Anti-platelet antibody assay was positive for antiplatelet IgG antibody. One week after extubation, the child suddenly developed features of raised intracranial 


\section{Persistent Thrombocytopenia Following Dengue Shock Syndrome}

pressure. Cranial non-contrast computerized tomogram (NCCT) was suggestive of massive intracranial bleed in the right temporo-parietal region with mid line shift; he soon herniated and died despite supportive care. Platelet count at the time of bleed was $70,000 / \mathrm{mm}^{3}$.

The AIIMS institute Ethics Committee waived off the need for informed consent for reporting this case.

\section{DISCUSSION}

This eleven-year-old boy had immune mediated and corticosteroid unresponsive prolonged thrombocytopenia after recovery from dengue shock syndrome (DSS), which led to massive intracranial bleed and his death.

While thrombocytopenia is the hallmark of dengue hemorrhagic fever (DHF), ${ }^{1}$ prolonged thrombocytopenia has been rarely reported in dengue patients. ${ }^{3,4}$ The mechanisms that contribute to pathogenesis of thrombocytopenia in dengue infection include immune mediated platelet destruction related to platelet associated immunoglobulin involving anti-dengue virus activity (PAIgG and PAIgM), ${ }^{6}$ virus-induced bone marrow suppression ${ }^{7}$ and increased binding of platelets to dengue virus infected endothelial cells. ${ }^{8}$ Our patient had negative DIC profile and blood cultures on multiple occasions and bone marrow examination revealed normalcy of all three cell lines. However, anti-platelet antibody assay was positive, suggesting immune origin of thrombocytopenia in this patient.

To the best of our knowledge, only two patients with dengue associated prolonged thrombocytopenia have been reported till date. ${ }^{3,4}$ One of these patients made a spontaneous recovery ${ }^{3}$ while the other one responded to corticosteroids. ${ }^{4}$ Intravenous immune globulin (IVIG) has been tried for dengue associated thrombocytopenia with some success, but the evidence is limited to a single case report. ${ }^{9}$ Our patient did not respond corticosteroids and daily PRP transfusion was required to keep the platelet count above $40,000 / \mathrm{mm}^{3}$. IVIG was not administered to our patient as the current evidence is not strong enough to justify the use of this expensive drug.

Our patient had massive intracranial bleed at a platelet count of $70,000 / \mathrm{mm}^{3}$. Though serious bleeding is rare in dengue patients, it is well known that platelet counts do not correlate very well with clinical bleeding, ${ }^{10}$ as was seen in this patient.

\section{CONCLUSION}

Prolonged thrombocytopenia is a rare but potentially devastating complication of DHF. Currently, little is known about its exact incidence, pathogenesis, outcome and therapy, and more research is required, but it would be prudent to monitor post recovery platelet counts in order to anticipate and prevent the serious complications related to this condition.

\section{REFERENCES}

1. Rigau-Perez JG, Clark GC, Gubler DJ, Reiter P, Sanders EJ, Vorndam AV. Dengue and dengue hemorrhagic fever. Lancet 1998; 352 : 971-977.

2. Ratageri VH, Shepur TA, Wari PK, Chavan SC, Mujahid IB, Yergolkar P. Clinical profile and outcome of dengue fever cases. Indian J Pediatr 2005; 72 : 705-706.

3. Kamil SM, Mohamad NH, Narazah MY, Khan FA. Dengue hemorrhagic fever with unusual prolonged thrombocytopenia. Singapore Med J 2006; 47 : 332-334.

4. Leong KW, Srinivas P. Corticosteroid-responsive prolonged thrombocytopenia following dengue hemorrhagic fever. Med J Malaysia 1993; $48:$ 369-372.

5. Nimmannitya S. Clinical manifestations of dengue/dengue hemorrhagic fever. In Monograph on dengue/dengue hemorrhagic fever. New Delhi; World Health Organization, 1993; 48-54.

6. Saito M, Oishi K, Inoue S, Dimaano EM, Alera MT, Robles AM et al. Association of increased platelet associated immunoglobulins with thrombocytopenia and severity of disease in secondary dengue virus infections. Clin Exp Immunol 2004; 138 : 299-303.

7. La Russa VF, Innis BL. Mechanism of dengue virus-induced bone marrow suppression. Bailliers Clin Hematol 1995; 8 : 249270.

8. Butthep P, Bunyaratvej A, Bhamarapravati N. Dengue virus and endothelial cell: a related phenomenon to thrombocytopenia and granulocytopenia in dengue hemorrhagic fever. Southeast Asian J Trop Med Public Health 1993; 24: 246-249.

9. Ascher DP, Laws HF, Hayes CG. The use of intravenous gammaglobulin in dengue fever, a case report. Southeast Asian J Trop Med Public Health 1989; 20 : 549-554.

10. Schexneider KI, Reedy EA. Thrombocytopenia in dengue fever. Curr Hematol Rep 2005; 4 : 145-148. 